\title{
The Current State of Business Intelligence and Analytics in Utah
}

\author{
John E. Anderson, Ph.D., Utah Valley University, janderson@uvu.edu \\ Jonathan Daniels, Utah Valley University, danielschoro@gmail.com \\ Daniel McDonald, Ph.D., Utah Valley University, daniel.mcdonald@uvu.edu \\ Richard Edvalson, Utah Valley University, rgedvalson@msn.com
}

\begin{abstract}
Business Intelligence and Analytics (BIA) has been adopted at an ever increasing pace by organizations. BIA is still evolving in maturity. This study compared new survey results collected from BIA professionals in Utah to a large 2011 survey conducted by SAS. We learned that most of the same BIA tools are being used, especially spreadsheets and reporting tools. BIA is used for business initiatives in sales, finance, strategy/planning, and marketing, and least in human resource management. BIA use has moved from isolated specific applications within an organization, to being more integrated. BIA use has also accelerated as shown by the change in use over the last 12 months with two-thirds citing their use of BIA increased moderately or significantly. Several of the biggest obstacles to BIA adoption and use were identified including data quality/integrity/consistency, lack of access to right data, and lack of appropriate BIA staff, among others.
\end{abstract}

Keywords: Business Intelligence, Analytics, Business Analytics

\section{INTRODUCTION}

Bloomberg and SAS conducted a large survey [1] to assess the status of business intelligence and analytics (BIA) in 2011 with 930 respondents. That study also compared the 2011 data to a similar study conducted in 2009 [5]. The researchers wanted to compare the SAS findings to the current status of BIA in organizations in Utah since it is a smaller geographic area with mostly small to medium sized businesses. It was hoped that the information gained would inform students interested in BIA careers of the current environment and status of the BIA profession as practiced in Utah. This research explored the status of business intelligence and analytics as practiced by organizations in Utah by collecting data through interviews and survey results.

\section{PROBLEM}

BIA [2] is a relatively new and quickly evolving part of many organization's information management strategies and has emerged as an important area of study for both practitioners and researchers. [3] There is great interest in but little knowledge of the current status of BIA in general, and in Utah more specifically. Many information systems students are interested BIA as a possible career choice. More information is needed for organizations to assess their current state of BIA maturity against those of other organizations. Information systems students also desire more information about how BIA is practiced in Utah and thus what skills and knowledge is needed by students to enter the BIA profession.

\section{METHOD}

The method of data collection for this study involved interviewing, using a structured questionnaire, 15 business intelligence/analytics professionals, and analyzing the data through both qualitative and quantitative techniques. Interviewees were invited via phone call or email to participate in a 45 minute interview during which they were asked questions from our questionnaire about the levels of adoption and use of business intelligence and analytics in their organizations. The questionnaire also included open-ended questions about professional background and if there is anything they feel should be added to the survey as it relates to their professional experience. Answers from the interviews were examined for emerging themes that show the level of business intelligence and analytics use. The participants were given and signed a consent form by the researcher. 


\section{FINDINGS}

The comparison of survey findings of the Utah 2013 data with the SAS 2011 data revealed interesting discoveries.

\section{BIA Tools Used}

The SAS 2011 study found that most organizations still rely on the old standard spreadsheet as the most used BIA tool. Likewise, as seen in Figure 1 spreadsheet software is still the most used BIA tool in the Utah 2013 survey.

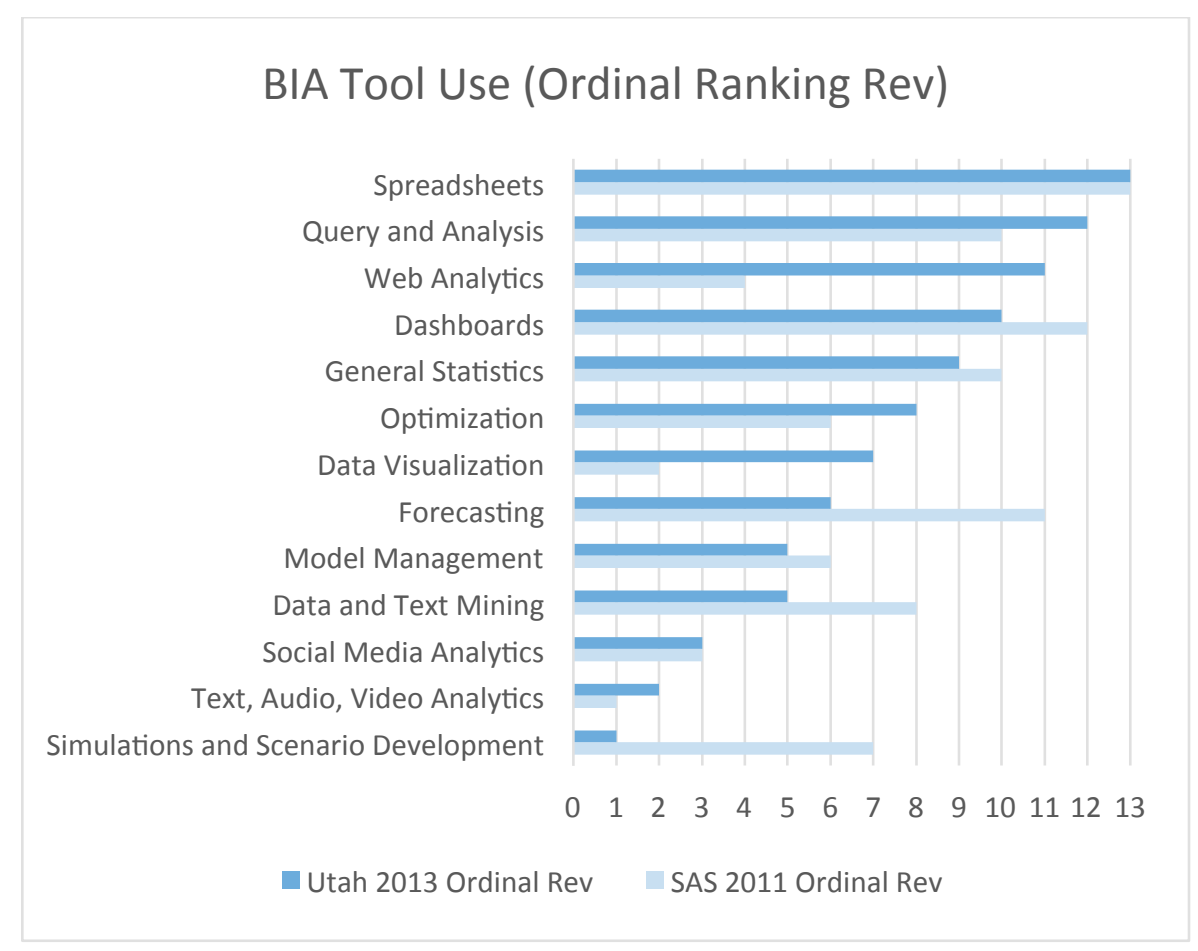

Figure 1 BIA Tool Use (Ordinal Ranking Rev)

At this point the data of the two surveys diverge a bit with the next four of the top five Utah 2013 tools being Query and Analysis, Web Analytics, Dashboards, and General Statistics; while the next four in the SAS 2011 data were Dashboards, Forecasting, Query and Analysis, and General Statistics. However, it is interesting to note that four of the top five tools are the same between both surveys, as seen in Table 1.

Table 1 BIA Tools Ordinal Ranking (Standard Competition Ranking)

\begin{tabular}{r|cc} 
& $\begin{array}{l}\text { Utah } \\
2013 \\
\text { Ordinal }\end{array}$ & $\begin{array}{l}\text { SAS 2011 } \\
\text { Ordinal }\end{array}$ \\
\hline Spreadsheets & 1 & 1 \\
Query and Analysis & 2 & 4 \\
Web Analytics & 3 & 10 \\
Dashboards & 4 & 2 \\
General Statistics & 5 & 4 \\
Optimization & 6 & 8 \\
Data Visualization & 7 & 12 \\
Forecasting & 8 & 3 \\
Data and Text Mining & 9 & 6 \\
Model Management & 9 & 8 \\
Social Media Analytics & 11 & 11 \\
Text, Audio, Video Analytics & 12 & 13 \\
Simulations and Scenario Development & 13 & 7
\end{tabular}




\section{BIA Business Initiatives}

The SAS 2011 study noted that organizations primarily use BIA in areas which rely on quantitative information to perform required analyses and predictions. The SAS study found that BIA was most used for Strategy/Planning, Finance, Marketing, and Information Technology. The Utah 2013 survey found that BIA was most used for Sales, Finance, Strategy/Planning, and Marketing as seen in Table 2 and Figure 2.

Table 2 BIA Business Initiatives

$\begin{array}{rcc} & \text { Utah } & \text { SAS } \\ & \begin{array}{c}2013 \\ \text { Ordinal }\end{array} & \begin{array}{c}2011 \\ \text { Ordinal }\end{array} \\ \text { Sales } & 1 & 5 \\ \text { Finance } & 2 & 2 \\ \text { Strategy/Planning } & 3 & 1 \\ \text { Marketing } & 3 & 3 \\ \text { Customer } & 5 & 7 \\ \text { Service/Support } & & \\ \text { Operations/Supply } & 6 & 6 \\ \text { Chain } & & \\ \text { Management } & & \\ \text { Information } & 7 & 4 \\ \text { Technology } & & \\ \text { Product } & 8 & 8 \\ \text { Development } & & \end{array}$

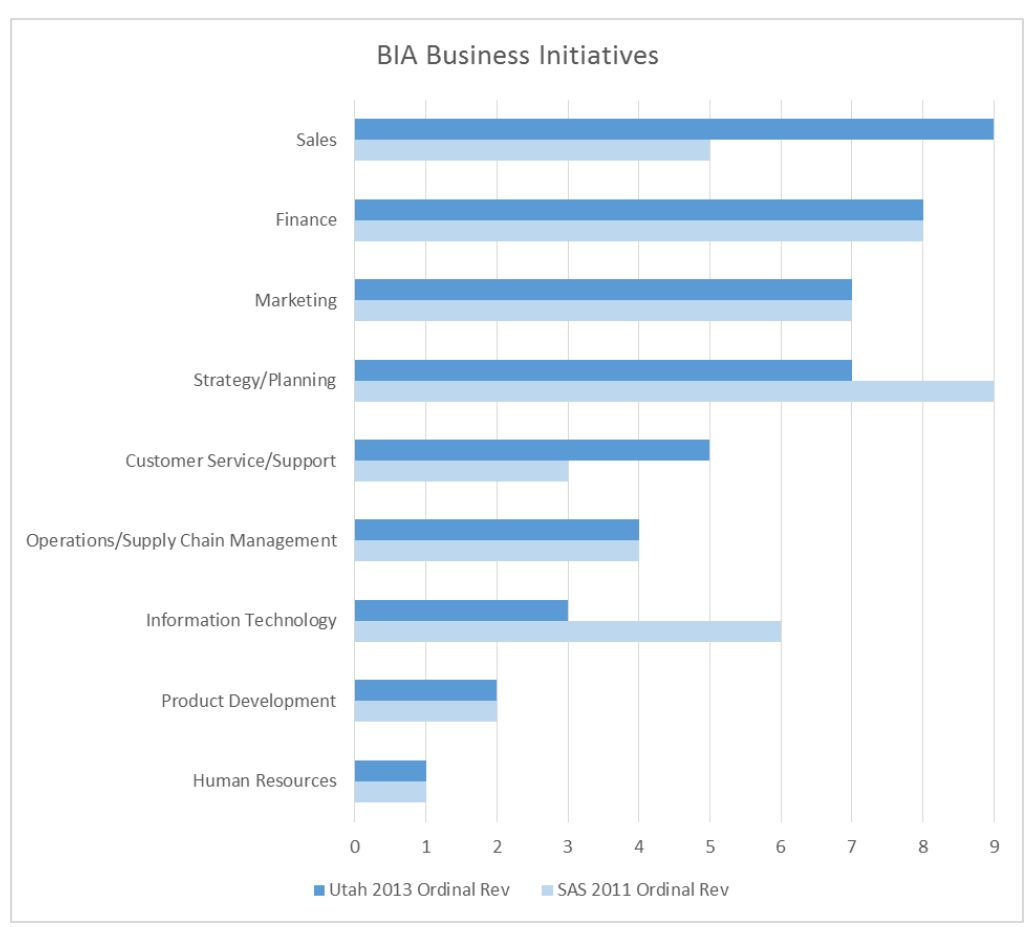

Figure 2 BIA Business Initiatives

\section{Use of BIA across Organization}

The SAS 2011 study found that organizational BIA use evolves incrementally through five stages beginning with not being used all. Secondly, BIA use begins in pilot programs and then advances to being used in specific functions. In the fourth stage, BIA use is incorporated into entire business units and finally in the fifth state, used by the entire organization. As seen in Figure 3, in the 2009 IDC study [4], the 2011 SAS study, and the 2013 Utah study most participants were in the "used in various business units" stage. What is interesting is the movement up the scale from the 2009 data to the 2013 data of organizational use of BIA. While the 2009 and 2011 studies showed still some organizations at the "not used in my organization" and "isolated use" stages, the 2013 data showed that all organizations were in the "used for specific functions" or higher stages. 


\section{Use of BIA Across Organization}

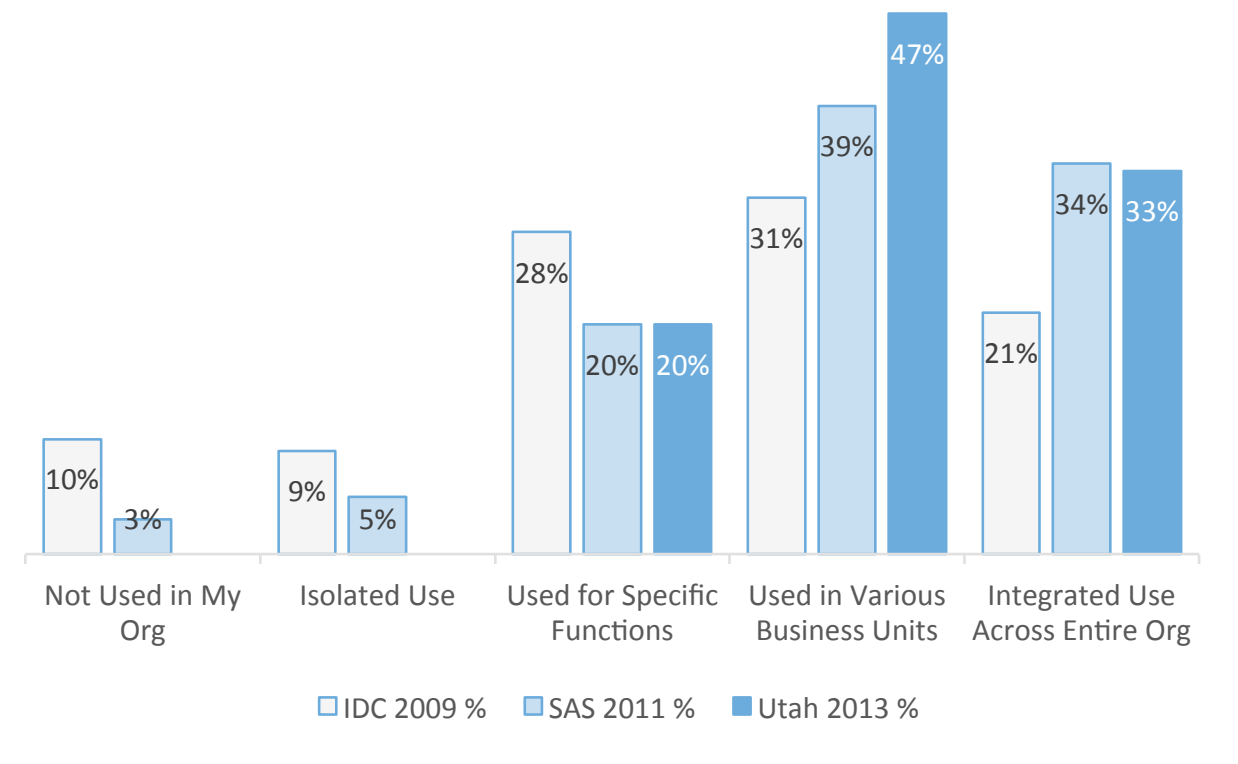

Figure 3 Use of BIA Across Organization

\section{Use of BIA Change in the last 12 Months}

The change in the use of BIA in organizations over the last 12 months also illustrates the acceleration of organizational use of BIA. In Figure 4 we see that while the 2011 SAS study participant organizations were in the "increased moderately" or "maintained" phases, the 2013 study participants moved up to mostly be in the "increased moderately" or "increased significantly" phases.

\section{Use of BIA Change in your Org Over Last 12 Months}

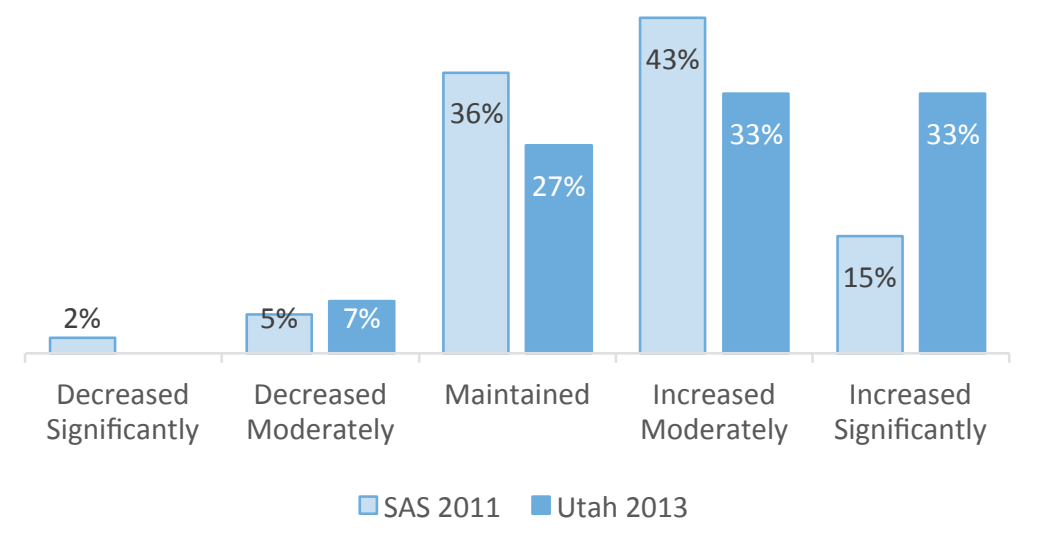

Figure 4 Use of BIA Change in Org Over Last 12 Months 


\section{BIA Obstacles}

The SAS 2011 study found that the biggest challenges in the adoption or use of BIA in organizations were the following: data quality/integrity/consistency, access to the right data, departmental silos, lack of knowledge of how to use BIA to make decisions, and lack of appropriate analytical staff. In Figure 5 and Table 3, we see that the Utah 2013 study participants responded with quite different BIA obstacles. The top five obstacles were the following: no single BIA solution that works for whole organization, a lack of appropriate BIA staff, a lack of BIA training, data quality/integrity/consistency, and users don't recognize power of BIA.

Table 3 BIA Obstacles

$$
\begin{array}{cc}
\text { Utah } & \text { SAS } \\
2013 & 2011 \\
\text { Ordinal } & \text { Ordinal }
\end{array}
$$

No Single BIA Solution that

Works for Whole Org

Lack of Appropriate BIA

Lack of BIA Training

Data Quality, Integrity, Consistency

Users Don't Recognize Power of BIA

Lack of Access to Right

Data

Managers Too Busy to Use BIA to Make Decisions BIA Expensive to Implement

Employees Don't Know How to Use BIA BIA Difficult to Use BIA Tools Difficult to Customize

BIA Expensive to Maintain BIA User Needs Difficult to Identify

Departmental Silos, Not Sharing Info Not Enough ROI on Current BIA Systems

$\begin{array}{ll}1 & \\ 2 & 5 \\ 2 & \\ 2 & 1 \\ 5 & \\ 5 & 2 \\ 7 & \\ 8 & \\ 9 & 4 \\ 10 & \\ 11 & \\ 12 & \\ 13 & \\ 13 & 3 \\ 15 & \end{array}$

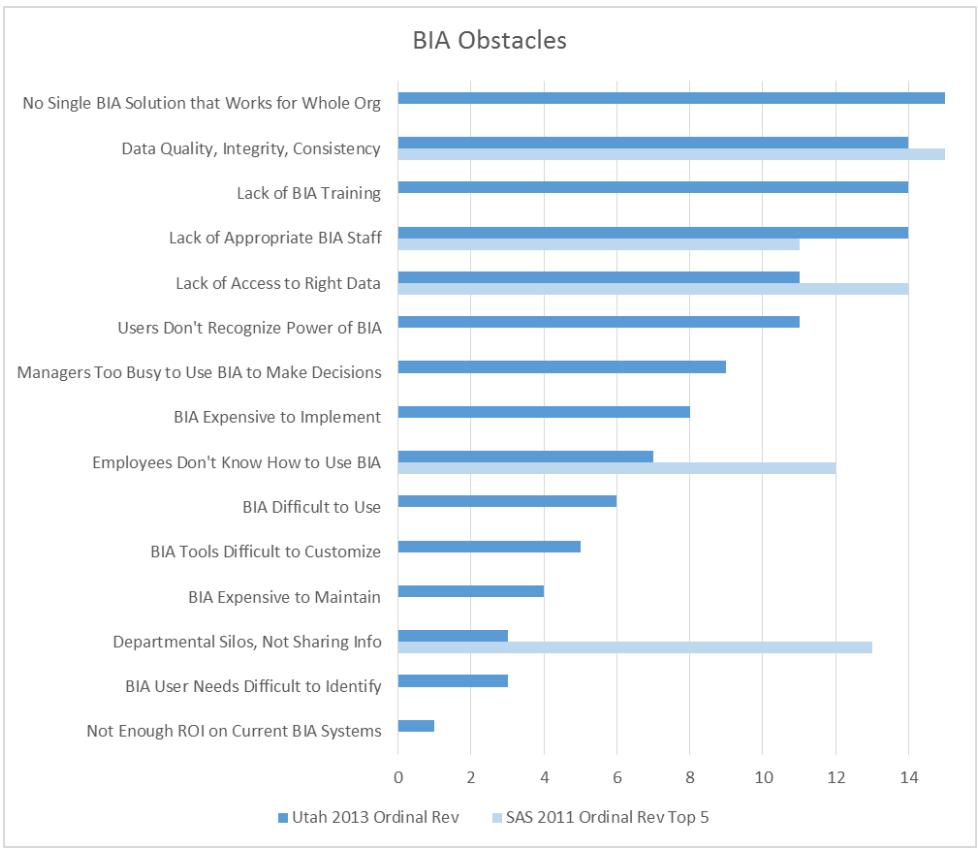

Figure 5 BIA Obstacles

\section{BIA Purposes}

We also asked respondents the reasons why they use BIA. As seen in Figure 6, the BIA purpose most important to respondents was to help plan for the future. The next most important BIA purpose was to help the organization to make better decisions. The BIA purposes respondents ranked third and fourth were to use BIA to better organize data and to anticipate trends. Using BIA to gain new knowledge based on past trends and to help make quick decisions were tied for fifth. 


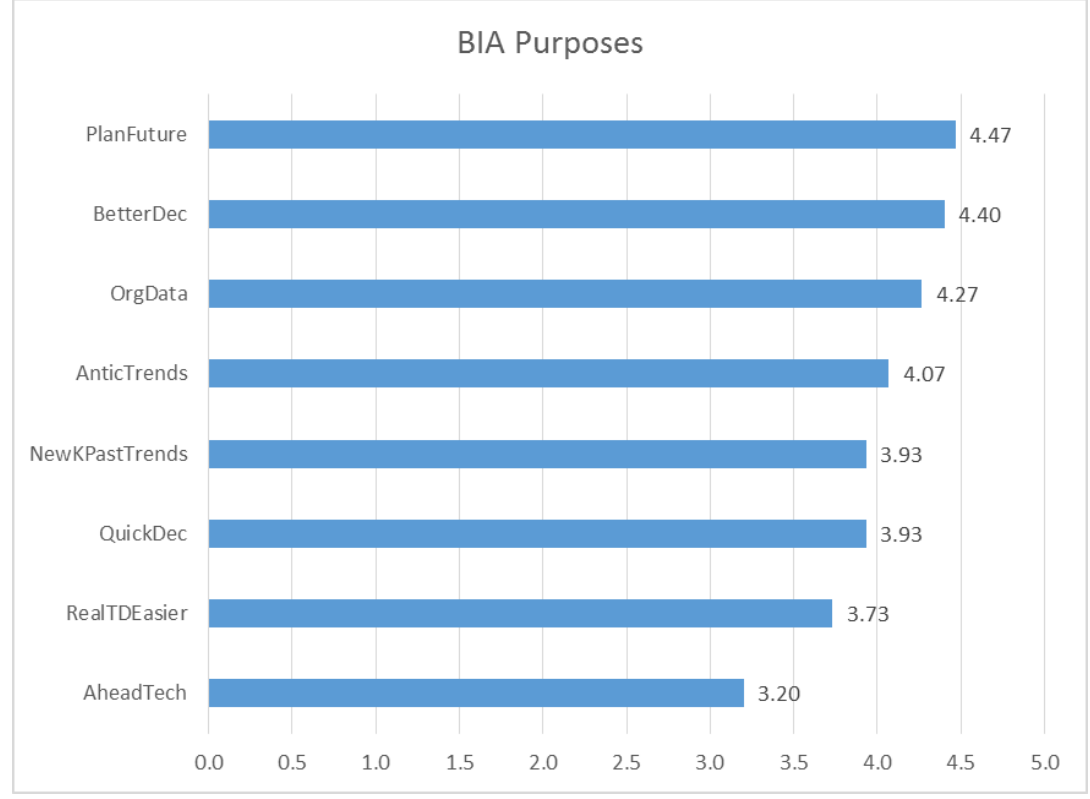

Figure 6 BIA Purposes

\section{BIA Organization Position}

We asked respondents whether the BIA function was centralized (ex. BIA has its own department), decentralized (ex. each of the various departments has a BIA position), or integrated in other roles (ex. other positions also do the BIA work, i.e. DBAs). Almost half characterized their organization's BIA function as Decentralized, another $40 \%$ characterized their organization's BIA function as centralized. About $40 \%$ also characterized their organization's BIA function as consisting of integrated positions. Also of interest is the number of respondents that viewed their organization's BIA activities in multiple ways as seen in Table 4. Approximately one-third consider their organization's BIA function as both centralized and decentralized, one-third as decentralized and integrated, onethird as centralized and integrated, and $27 \%$ as centralized-decentralized-integrated.

Table 4 BIA Organizational Position

\begin{tabular}{|lcc|} 
BIA Org Position & Number & Percent \\
\hline Centralized & 6 & $40 \%$ \\
Decentralized & 7 & $47 \%$ \\
Integrated & 6 & $40 \%$ \\
\hline Centralized \& Decentralized & 5 & $33 \%$ \\
Decentralized \& Integrated & 5 & $33 \%$ \\
Centralized \& Integrated & 5 & $33 \%$ \\
Centralized \& Decentralized \& Integrated & 4 & $27 \%$ \\
\hline
\end{tabular}

\section{DISCUSSION}

When we compare this study's findings to that of the SAS 2011 study we see both similarities and differences. The similarities of BIA tool use are striking in that four of the five most used BIA tools (spreadsheets, query and analysis, dashboards, and general statistics) are the same (though in a different order). The differences in the top five tools, forecasting for the SAS 2011 study and web analytics for the Utah 2013 study, show a possible difference in 


\section{Issues in Information Systems \\ Volume 15, Issue II, pp. 359-366, 2014}

BIA sophistication. The SAS 2011 respondents are from the largest organizations with the most knowledgeable BIA staffs. The rankings of the other tools illustrate this difference in sophistication in a more pronounced way with the SAS 2011 next most used tools being "data and text mining" and "simulations and scenario development" while the Utah 2013 next most used tools being "optimization" and "data visualization." Data and text mining and simulations are sophisticated tools used by larger organizations. The tools used most by the Utah 2013 participants are focused on reporting rather than predictive analytics or higher order BIA. Data visualization is currently one of the most popular BIA reporting tools with many new "user friendly" BIA data visualization tools for even smaller organizations. Optimization is also achievable by smaller organizations, especially in the marketing and logistics functions. The fact that data mining, forecasting, and social media analytics is still unused by many Utah organizations shows a focus on more mature reporting tools and a lack of sophistication with higher level BIA tool use.

In addition, in contrast to the SAS study, the Utah study shows a focus on web analytics. Utah was home to a leading web analytics company, namely Omniture. Omniture was acquired by Adobe in 2009 for $\$ 1.8$ billion. Having such a large analytics firm local, likely influenced the adoption and visibility of web analytics tools. In addition, because Omniture stored all the data and provided the tools online, companies could incorporate web analytics with limited in house expertise. Such a solution catered to the smaller to medium sized companies in the Utah market.

There were also strong similarities between both the 2011 and 2013 studies in terms of use of BIA in business initiatives. In both studies, four of the five most prominent uses of BIA were the same, namely finance, marketing, strategy/planning, and sales (though in different orders of use). In the Utah study, Sales was the top business initiative for BIA, while it was fifth in the SAS study. This difference can in part be connected to the high use of web analytics in the Utah study. Often the focus of web analytics is to increase customer sales, especially for webfocused retail companies like Overstock and Backcountry.

The SAS 2011 study top five business initiatives included information technology while the Utah 2013 top five included customer service/support. This difference may be a difference in organizations surveyed or a trend to more BIA use in the customer service function and less in the IT function. Non top five similarities were also prominent. Data from both studies showed operations/supply chain management at sixth, product development at eighth, and human resources at ninth (or last). Product development and human resources are functions that probably do not utilize BIA to the extent as other functions. Possible reasons include lack of budget, or a lack of perceived need for analytical information given the common decisions faced in HR.

By comparing the IDC 2009 and the SAS 2011 and the Utah 2013 data on the question of use of BIA across the organization illustrates the evolving use of BIA over the past four years. The use of BIA has moved further toward integrated use across the entire organization. Approximately $80 \%$ are using BIA either integrated across the entire organization or in various business units. Only about $20 \%$ are using BIA for specific functions and almost none for an isolated very specific use. BIA has become mature in the sense of extensive use (at least at some level) in most of the organization. The acceleration of organizational use of BIA is also shown by the change in the use of BIA in organizations over the last 12 months with two-thirds citing the use of BIA increased moderately or significantly.

There are mostly similarities in the biggest challenges or obstacles in the adoption or use of BIA in organizations between the SAS 2011 and Utah 2013 studies. There is overlap in three of the top five obstacles: data quality/integrity/consistency, lack of access to right data, and lack of appropriate BIA staff. Data quality and lack of appropriate BIA staff is probably due to the lack of well-trained BIA professionals. Both studies also agree on the importance of the obstacle of managers too busy to use BIA to make decisions or don't know how to use BIA to make decisions. This could be due to lack of BIA training or users not understanding the power of BIA to aid decision-making. One study pointed out the need for shared knowledge among the strategic and operational levels as possible drivers of BI business value. [4] The differences between the studies is also instructive. The biggest obstacle in the Utah 2013 survey is that no company feels that there is one single BIA solution for the entire organization. This difference between the studies is interesting. It seems those study participants in Utah would like a more centralized approach, but find the technology lacking. Perhaps this obstacle shows a greater exposure to BIA tools over the years since the 2011 study and find the current solutions to still be lacking. It appears customers and vendors disagree as to the state of VIA tools. Vendors claim to offer solutions for the entire organization, and yet customers feel that there is not one solution for their entire company. A deficiency in the BIA tools could explain 
why study participants see the BIA function as being both centralized and decentralized. If and when tools can be more general purpose, perhaps the BIA function can be more centralized. A top five obstacle in the SAS 2011 study that was found much lower in the Utah 2013 study is the obstacle of departmental silos, not sharing information. A related obstacle is lack of access to the right data, but the departmental silos obstacle also adds the dimensions of lack of integrated processes and not sharing data consistently. Perhaps the change between the 2011 and 2013 surveys is that more systems have been integrated in more organizations. The fact that ROI is the smallest obstacle shows that companies believe in and expect returns on BIA investments.

The purposes organizations use BIA for are to help plan for the future, to help the organization to make better decisions, to better organize data, and to anticipate trends. It is interesting that while most organizations primarily implement the reporting functions of BIA, which focus on past historical data, organizations use these BIA reports for future planning and trend spotting. The organizations also use the BIA reporting function to better organize data, perhaps making it easier to consume. Lower in the ranking we find that organizations use BIA to gain new knowledge based on past trends and to help make quick decisions. Possibly here we see reflected the BIA predictive analytics function and real-time decision making function, though less mature, still growing in importance.

The BIA function is decentralized in about half of organizations, and centralized in just under half of organizations responding to the survey. While the literature has often argued that a centralized BIA function is preferred, perhaps the lag is just a level of immaturity. Or is decentralization preferred by practitioners, giving more control to departments. Just under half also characterize their BIA positions as integrated, meaning that the other positions also do BIA functions, such as a DBA. Not having dedicated BIA staff shows that BIA is still immature in many organizations. However, many organizations characterize their BIA functions in multiple ways in a kind of splitpersonality such as centralized and decentralized and integrated at the same time. Perhaps organizations have a central BIA function to gain and share access to large computing resources such as a data warehouse etc. Organizations also however, at the same time, need BIA deep domain knowledge to be able to create the kind of intelligence needed to improve performance. In addition, the integrated nature of the BIA function could be leftover part of the evolving nature of BIA as it has often been implemented in the past in a focused piece-meal way.

\section{CONCLUSIONS}

This research explored the status of business intelligence and analytics as practiced by organizations in Utah by collecting data through interviews and survey results. The survey results were compared to an earlier study conducted by Bloomberg and SAS in 2011. The similarities and differences in the survey results show the further evolution of BIA use in organizations, especially in a geographic region of mostly small to medium organizations. We also added some knowledge of organizational dimensions of BIA to the literature. This information would also be valuable to students wanting to enter the BIA field by delineating the skills and knowledge currently practiced in the BIA profession in Utah.

\section{REFERENCES}

1. Bloomberg BusinessWeek Research Services and SAS (2011). The current state of business analytics: Where do we go from here? White Paper available at http://www.sas.com/reg/gen/corp/1504633

2. Chaudhuri, S., \& Dayal, U., \& Narasayya, V. (2011). An overview of business intelligence technology. Communications of the ACM 54(8), pp 88-98.

3. Chen, H., \& Chiang, R., \& Storey, V., (2012). Business intelligence and analytics: from big data to big impact. MIS Quarterly 36(4), pp. 1165-1188/December 2012

4. Elbashir, M., \& Collier, P., \& Sutton, S., \& Davern, M., \& Leech, S. (2013) Enhancing the business value of business intelligence: the role of shared knowledge and assimilation. Journal of Information Systems 27(2), pp 87-105

5. International Data Corporation, "IDC's Worldwide Business Intelligence Tools Tracker Finds the Market Surpassed \$4 Billion in Revenues in the Second Half of 2010 with Further Growth Expected in 2011," news release, June 27, 2011, http://www.businesswire.com/news/ home/20110627005197/en/IDCsWorldwide-Business-Intelligence-Tools-Tracker-Finds. 